\title{
IR-780-loaded polymeric micelles enhance the efficacy of photothermal therapy in treating breast cancer lymphatic metastasis in mice
}

\author{
Bin $\mathrm{HE}^{1}$, Hai-yan $\mathrm{HU}^{1}$, Tao TAN², Hong WANG ${ }^{2}$, Kao-xiang $\mathrm{SUN}^{1, *}$, Ya-ping $\mathrm{LI}^{2}$, Zhi-wen ZHANG ${ }^{2, *}$ \\ ${ }^{1}$ School of Pharmacy, Collaborative Innovation Center of Advanced Drug Delivery System and Biotech Drugs in Universities of \\ Shandong, Key Laboratory of Molecular Pharmacology and Drug Evaluation (Yantai University), Ministry of Education, Yantai University, \\ Yantai 264005, China; ${ }^{2}$ State Key Laboratory of Drug Research \& Center of Pharmaceutics, Shanghai Institute of Materia Medica, \\ Chinese Academy of Sciences, Shanghai 201203, China
}

\begin{abstract}
Cancer metastasis is responsible for over $90 \%$ of breast cancer-related deaths, and inhibiting lymph node metastasis is an option to treat metastatic disease. Herein, we report the use of IR-780-loaded polymeric micelles (IPMs) for effective photothermal therapy (PTT) of breast cancer lymphatic metastasis. The IPMs were nanometer-sized micelles with a mean diameter of $25.6 \mathrm{~nm}$ and had good stability in simulated physiological solutions. Under 808-nm laser irradiation, IPMs exhibited high heat-generating capability in both in vitro and in vivo experiments. After intravenous injection, IPMs specifically accumulated in the tumor and metastatic lymph nodes and penetrated into these tissues. Moreover, a single IPMs treatment plus laser irradiation significantly inhibited primary tumor growth and suppressed lymphatic metastasis by $88.2 \%$. Therefore, IPMs are an encouraging platform for PTT applications in treatment of metastatic breast cancer.
\end{abstract}

Keywords: cancer metastasis; lymphatic metastasis; IR-780; polymeric micelles; photothermal therapy

Acta Pharmacologica Sinica (2018) 39: 132-139; doi: 10.1038/aps.2017.109; published online 10 Aug 2017

\section{Introduction}

Cancer metastasis, rather than the primary tumor, is the leading cause of the high mortality of breast cancer patients ${ }^{[1-5]}$. Metastasis refers to the spread of cancer cells from the primary tumor into the circulatory system and colonization at distant $\operatorname{organs}^{[2,3,6]}$. During the metastatic process, lymph nodes near the primary tumor are usually the most frequent sites where cancer cells are disseminated ${ }^{[6-10]}$. Accordingly, inhibiting lymphatic metastasis in breast cancer is an option for treating metastatic disease.

Photothermal therapy (PTT) is a newly developed therapeutic modality in cancer treatment, which has attracted increasing attention in treating metastatic cancer ${ }^{[11-17]}$. In PTT, near-infrared (NIR) laser absorbing agents can be used to generate hyperthermia with NIR laser irradiation for thermal ablation of cancer cells. PTT thus has the unique advantages

\footnotetext{
*To whom correspondence should be addressed.

E-mail sunkaoxiang@luye.cn (Kao-xiang SUN); zwzhang0125@simm.ac.cn (Zhi-wen ZHANG)

Received 2017-04-24 Accepted 2017-05-19
}

of minimal invasiveness, high specificity and favorable efficiency $^{[12]}$. Many organic and inorganic nanomaterials have been designed for PTT application, but few nano-agents have been explored for treating lymphatic metastasis ${ }^{[12,18,19]}$. However, most current photothermal nano-agents are inorganic nanomaterials that are administered by local injection. Hence, their further application is restricted, owing to their nonbiodegradable nature, potential long-term toxicity and limited accumulation in metastatic lymph nodes ${ }^{[8,19-21]}$. Therefore, photothermal nano-agents should be rationally designed to improve their potential applications of PTT for breast cancer lymphatic metastasis.

Recently, the NIR fluorescent dyes and their nano-agents have attracted increasing attention in PTT applications ${ }^{[7,11,13]}$. Typically, IR-780, a hydrophobic NIR fluorescence dye with a maximum absorbance at $780 \mathrm{~nm}$, has strong light-absorbing abilities, thus making it an ideal candidate for PTT applications ${ }^{[22-24]}$. However, IR-780 is highly insoluble in water and readily aggregates in physiological solutions, thereby leading to inefficient heat-generating effects under NIR laser irradiation and interfering with PTT. To circumvent these draw- 
backs, IR-780 can be loaded into nano-assemblies to improve the potential for PTT applications. However, the possible application of IR-780 loaded nano-agents to treat lymphatic metastasis in breast cancer has not yet been explored.

Herein, we report IR-780 loaded polymeric micelles (IPMs) with efficient tumor and lymph targeting abilities in for breast cancer lymphatic metastasis. IPMs were generated from 1,2-distearoyl-sn-glycero-3-phosphoethanolamine$N$-methoxy(polyethylene glycol)-2000 (DSPE-PEG2000) and IR-780 through a self-assembly technique. The in vitro physicochemical properties of the IPMs were characterized. The specific accumulation of IPMs in tumor and metastatic lymph nodes was evaluated in a lymphatic metastatic breast cancer model. Moreover, the in vivo therapeutic efficacy was evaluated to validate the effects on inhibiting lymphatic metastasis in breast cancer.

\section{Materials and methods Materials}

DSPE-PEG2000 was supplied by Shanghai Advanced Vehicle Technology Pharmaceutical Ltd (Shanghai, China). IR-780 iodide was obtained from Sigma-Aldrich (Shanghai, China). The 4T1 cancer cells were provided by the Shanghai Cell Bank, Chinese Academy of Science (CAS). Cells were cultured in RPMI-1640 containing 10\% fetal bovine serum (FBS, Gibco) at $37{ }^{\circ} \mathrm{C}$ in a humidified and $5 \% \mathrm{CO}_{2}$ incubator.

Nude mice (18-20 g, female) were provided by the Shanghai Laboratory Animal Center, CAS, and maintained in the animal care facility for acclimatization at least $3 \mathrm{~d}$ prior to the experiment. The lymph metastatic breast cancer model was induced by the subcutaneous injection of $5 \times 10^{5} 4 \mathrm{~T} 1$ cells into the footpad in nude mice. The in vivo evaluations of IPMs in the lymphatic metastatic tumor-bearing mice were performed $12 \mathrm{~d}$ after the inoculation. The experiments were approved by the Institutional Animal Care and Use Committee (IACUC) of Shanghai Institute of Materia Medica, CAS.

\section{Preparation of IPMs}

IPMs were prepared from DSPE-PEG and IR-780 through a self-assembly technique. In brief, DSPE-PEG2000 and IR-780 were dissolved in $1.0 \mathrm{~mL}$ of methanol and mixed by vortexing in a round flask. Then, the mixed solution was evaporated to dryness to form a thin film under reduced pressure (Heidolph Laborata 4000, Germany). Afterward, the thin film was dispersed in $1.0 \mathrm{~mL}$ of phosphate buffered solution (PBS, $\mathrm{pH}$ 7.4) by gentle shaking at room temperature. IPMs spontaneously formed, and the final concentration of IR-780 was 0.15 $\mathrm{mg} / \mathrm{mL}$. As a control, blank micelles of DSPE-PEG without IR-780 were prepared through the same procedure.

\section{Characterization of IPMs}

The IPMs morphology was detected under a field emission transmission electronic microscope (FE-TEM) (Tecnai G2 F20 S-Twin, FEI). Briefly, IPMs were diluted, negatively stained with $2 \%$ phosphotungstic acid solution (w/v, $\mathrm{pH} 5.8)$, and loaded on a copper grid. Then, the grid was rinsed with dis- tilled water and dried under room temperature for further imaging under FE-TEM. The size distribution was evaluated by image analysis. Meanwhile, to avoid the interference of IR-780 in IPMs in the analysis by dynamic light scattering (DLS), the size distribution and zeta potential values of blank IPMs without IR-780 were measured on a Zetasizer Nano ZS 90 instrument (Malvern, UK).

Moreover, the encapsulation efficiency (EE) and drug loading of IR-780 in IPMs were determined via an ultrafiltration method (molecular cut-off, 3000 Da, Millipore). Free IR-780 was separated from IPMs by centrifugation at $12000 \times g$ for 30 min (Biofuge Stratos, Thermo). The IR-780 level in the filtrate and IPMs was measured with a fluorescence spectrophotometer (Thermo Fisher Scientific Oy Ratastie2, FI-01620 Vantaa, Finland) for subsequent calculations.

\section{In vitro stability measurements}

To determine the stability of IPMs in the mimicked physiological solutions, IPMs were diluted 10 times with water, PBS (pH 7.4) and PBS (pH 7.4) containing 10\% FBS $(15 \mu \mathrm{g} / \mathrm{mL}$ of IR-780); then, they were incubated at $37^{\circ} \mathrm{C}$ for $24 \mathrm{~h}$. At predetermined time intervals, the encapsulation efficiency of IR-780 in IPMs was evaluated to determine the stability over time. The EE of IR-780 in IPMs was measured according to the methods described above. Moreover, the fluorescence intensities of these samples were analyzed with a fluorescence spectrometer (F4600, HITACHI, Japan) at specific time intervals.

\section{In vitro photothermal efficiency}

To evaluate the in vitro heat-generating capability, IPMs and free IR-780 in PBS ( $\mathrm{pH} 7.4$ ) (at $0.15 \mathrm{mg} / \mathrm{mL}$ ) were used for the evaluations. For the preparation of free IR-780 in PBS $(\mathrm{pH}$ 7.4), $100 \mu \mathrm{L}$ of free IR-780 in methanol $(1.5 \mathrm{mg} / \mathrm{mL})$ was mixed with $900 \mu \mathrm{L}$ of PBS (pH 7.4). Meanwhile, $100 \mu \mathrm{L}$ of free IR-780 in methanol $(1.5 \mathrm{mg} / \mathrm{mL})$ was diluted with methanol to prepare free IR-780 in methanol at $0.15 \mathrm{mg} / \mathrm{mL}$ as control. Typical IPMs, free IR-780 in PBS (pH 7.4) and free IR-780 in methanol were photographed. Then, the absorption spectra of IPMs, free IR-780 in PBS (pH 7.4) and free IR-780 (1.5 $\mu \mathrm{g} / \mathrm{mL})$ were analyzed with a UV-visible spectrophotometer (UV-2450, Shimadzu, Japan).

Then, the thermal images and temperature changes of IPMs were recorded using an infrared thermal camera (A150-15-M, Irtech Ltd). The saline control, free IR-780 suspension and IPMs $(0.15 \mathrm{mg} / \mathrm{mL}$ of IR-780) were exposed to $808 \mathrm{~nm}$ laser irradiation (MDL-N-10W, Changchun New Industries, China) at a power density of $3.0 \mathrm{~W} / \mathrm{cm}^{2}$ for the measurements. Thermal images were captured, and temperature changes were recorded.

\section{In vivo distribution of IPMs in a lymph metastatic breast cancer} model

The in vivo distribution of IPMs was detected in a lymph metastatic breast cancer model by using an in vivo imaging system (IVIS Spectrum, Perkin-Elmer). The lymph metastatic breast cancer model was developed as described above. The 
lymphatic metastasis was detected as swelled lymph nodes at $12 \mathrm{~d}$ after inoculation. IPMs were administered to the tumorbearing mice via tail vein injection at $1.5 \mathrm{mg} / \mathrm{kg}$ IR-780. At specific time intervals, mice were anesthetized with pentobarbital sodium and imaged under the in vivo imaging system. At $4.0 \mathrm{~h}$ after injection, mice were autopsied, and the major organs, including the heart, liver, spleen, lungs, kidneys, tumor and lymph nodes, were carefully removed. These samples were rinsed with PBS (pH 7.4) and analyzed under the in vivo imaging system. By contrast, mice without any treatment underwent the same procedure and were imaged as the negative control.

In vivo tumor and lymphatic metastasis targeting visualized with a laser confocal scanning microscope (LCSM)

The in vivo tumor and lymphatic metastasis targeting of IPMs was further measured under LCSM (Fluoview FV 1000, Olympus, Japan). IPMs were intravenously administered in the lymph metastatic breast cancer model at $1.5 \mathrm{mg} / \mathrm{kg}$ IR-780. At $4.0 \mathrm{~h}$ after injection, the tumors and metastatic lymph nodes were carefully collected for detection. These samples were rinsed with PBS (pH 7.4) and frozen in cryoembedding medium (OCT) for subsequent sectioning at $15 \mu \mathrm{m}$ (CM1950, Leica). These sections were mounted onto glass slides and stained with DAPI (blue, Beyotime) and phalloidin-FITC (green, Beyotime) according to the manufacturer's protocols. Afterwards, the sections were observed under a LCSM, and the fluorescence signals of IPMs were recorded to examine the distribution in these organs, especially in tumor tissues and lymph nodes.

\section{In vivo PTT of lymphatic metastasis in breast cancer}

The inhibitory effects of IPMs on primary tumor growth and lymphatic metastasis was measured in lymph metastatic breast cancer models. The tumor-bearing mice were developed as described above. At $6 \mathrm{~d}$ after inoculation, mice were randomly divided into four groups $(n=5)$ and were respectively treated with a single treatment of saline control, saline+Laser, IPMs and IPMs+Laser at $1.5 \mathrm{mg} / \mathrm{kg}$ IR-780. At $4.0 \mathrm{~h}$ after injection, the tumor tissues from saline+Laser and IPMs+Laser treated groups were exposed to $808 \mathrm{~nm}$ laser at $3 \mathrm{~W} / \mathrm{cm}^{2}$ for $5 \mathrm{~min}$. The temperature variations and thermal images of the tumor were detected under an infrared thermal camera. At d 12 after laser irradiation, the primary tumor and metastatic lymph nodes from each group were carefully collected, photographed and weighted to calculate the inhibition rate on tumor growth and lymphatic metastasis of breast cancer. Afterward, the metastatic lymph nodes were evaluated by histological examinations with H\&E staining to confirm the lymphatic metastasis. The metastatic lesions presented as cell clusters with darkly stained nuclei. In addition, the body weights of the tumor-bearing mice were recorded every three days.

\section{Statistical analysis}

All results are presented as the mean \pm standard deviation (SD).
Statistical analysis was performed using two-tailed Student's $t$-tests. The differences were considered significant at $P<0.05$.

\section{Results and Discussion}

\section{Preparation and characterization of IPMs}

IPMs were prepared from IR-780 and DSPE-PEG2000 through a self-assembly technique. The IPMs morphology was visualized with FE-TEM (Figure 1A), which showed that IPMs were homogenous nanometer-sized particles. The IPMs particle size distribution was further assessed by image analysis. Statistical image analysis of more than 40 individual IPMs indicated that the average IPMs particle size was $25.6 \pm 4.0 \mathrm{~nm}$. Owing to the interference of IR-780 with the DLS analysis, the size distribution of blank micelles without IR-780 was evaluated (Figure 1B). The blank micelles had a hydrodynamic diameter of $15.3 \pm 5.9 \mathrm{~nm}$ with a PDI of 0.154 , and they had a zeta potential value of $-5.1 \pm 0.8 \mathrm{mv}$. Considering the vital role of the particle size in tumor targeting, the small IPMs size may be promising for targeting lymph nodes in breast cancer ${ }^{[25-28]}$. Moreover, the IPMs encapsulation efficiency and drug loading capacity of IR-780 were determined by fluorescence analysis. The measured results indicated that the IPMs EE of IR-780 was $95.17 \% \pm 0.08 \%$, and the drug loading capacity was $1.40 \% \pm 0.01 \%$. These experimental results suggested the high encapsulation of IR-780 in the small IPMs.

Then, IPMs were incubated with distilled water, PBS at $\mathrm{pH}$ 7.4 and PBS (pH 7.4) with $10 \%$ FBS at $37^{\circ} \mathrm{C}$ (with $15 \mu \mathrm{g} / \mathrm{mL}$ IR-780) to evaluate their stability in the physiological solutions. At predetermined time intervals, the EE values of IR-780 in IPMs were measured to assess the stability. When IPMs were incubated with PBS at $\mathrm{pH} 7.4$ and PBS (pH 7.4) with $10 \%$ FBS, the EE values of IR-780 in IPMs were $95.69 \% \pm 0.11 \%$ and $95.50 \% \pm 0.12 \%$, respectively, values comparable to that in distilled water. As shown in Figure 1C, the EE values of IPMs in these three media had scarcely changed at 4.0 and $24.0 \mathrm{~h}$ after dilution. Moreover, the fluorescence intensities of IPMs in these media were measured (Figure 1D). When IPMs were incubated with PBS (pH 7.4) and PBS (pH 7.4) with 10\% FBS, the fluorescence intensity of IPMs was scarcely changed compared with that in water. In addition, the fluorescence intensity of IPMs was not changed at $4.0 \mathrm{~h}$ or $24.0 \mathrm{~h}$ after incubation (Figure 1D). These experimental results suggested good stability of IPMs in these physiological solutions over time; thus IPMs are suitable for further in vivo evaluations.

Typical photographs of IPMs, free IR-780 in methanol and free IR-780 in PBS (pH 7.4) $(0.15 \mathrm{mg} / \mathrm{mL}$ of IR-780) showed that IR-780 was easily precipitated in PBS (pH 7.4), but no precipitation was detected in IPMs and IR-780 in methanol (Figure 2A). The precipitation of IR-780 in PBS ( $\mathrm{pH} 7.4$ ) may have been due to the poor water-solubility, thus suggesting that the free IR-780 suspension could not be used for in vivo measurements. Then, the absorption profiles of these samples at $1.5 \mu \mathrm{g} / \mathrm{mL}$ IR-780 were detected (Figure 2B). The free IR-780 in PBS (pH 7.4) exhibited negligible absorption in the NIR regions (Figure 2B). However, when IR-780 was loaded into the polymeric micelles, IPMs showed strong absorption in 
A

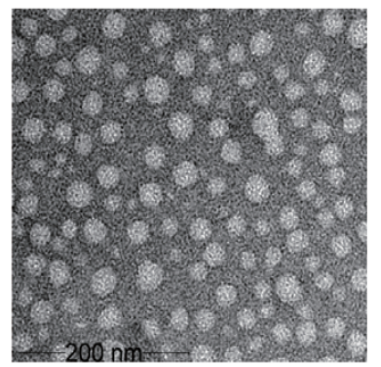

C

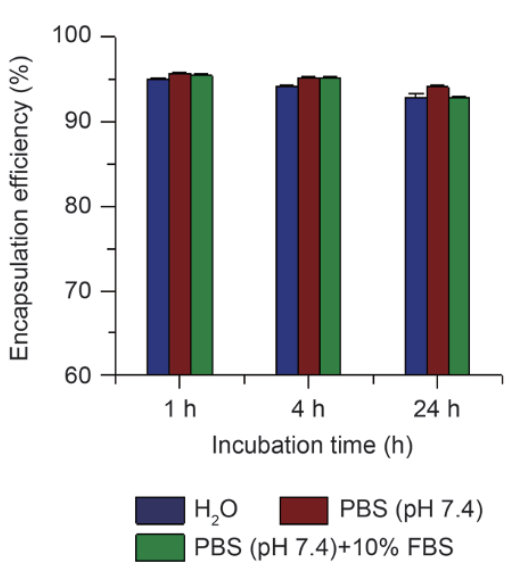

B
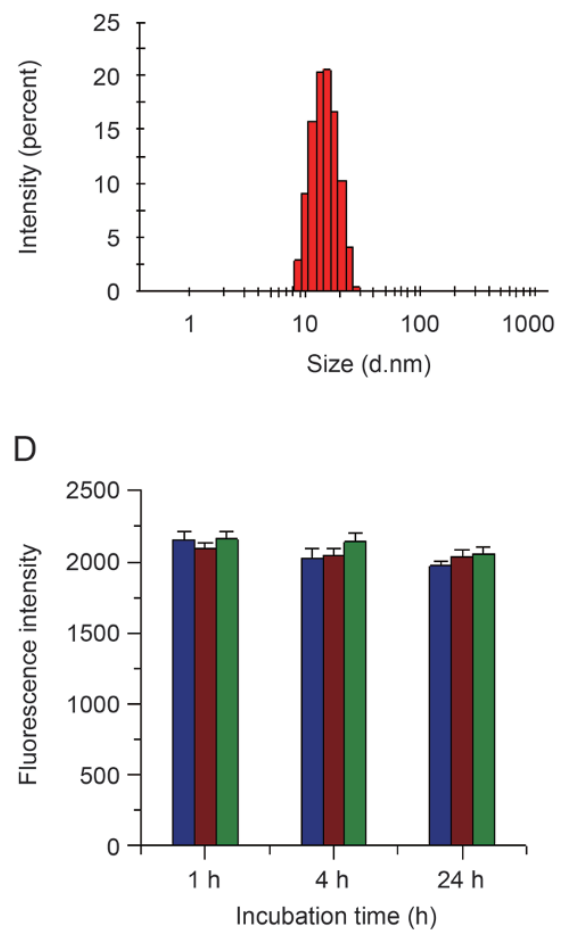

$\mathrm{H}_{2} \mathrm{O}$ PBS (pH 7.4) PBS (pH 7.4)+10\% FBS

Figure 1. The in vitro characterizations of IPM. (A) The typical FE-TEM images of IPM; (B) The size distribution of blank micelles without IR-780 measured by DLS analysis; (C) the stability of IPM in different media at $37^{\circ} \mathrm{C}$ characterized by the encapsulation efficiency of IR-780; (D) the stability of IPM in different media at $37^{\circ} \mathrm{C}$ characterized by the fluorescence intensity.

A

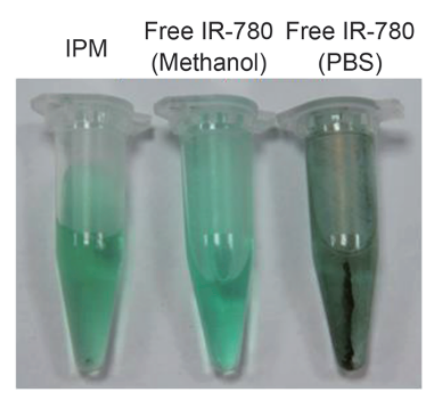

C

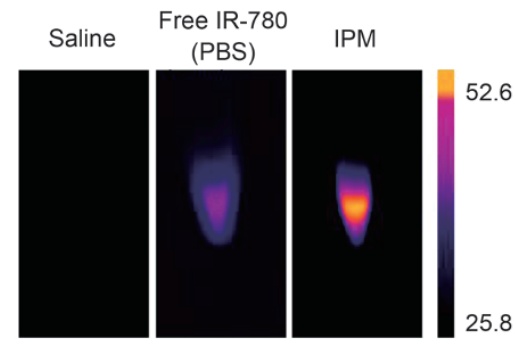

B

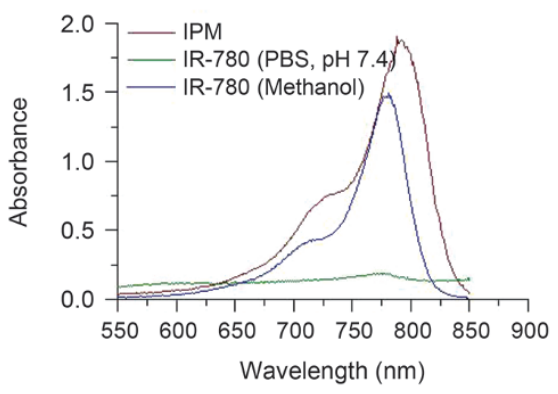

D

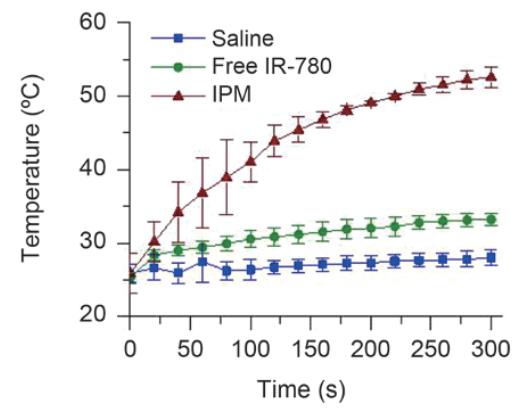

Figure 2. The in vitro photothermal characterizations of IPM. (A) The photograph of IPM, free IR-780 in methanol and free IR-780 in PBS (pH 7.4 ) $(0.15 \mathrm{mg} / \mathrm{mL}$ of IR-780); (B) the absorption profiles of IPM, free IR-780 in methanol and free IR-780 in PBS (pH 7.4) (1.5 $\mu \mathrm{g} / \mathrm{mL}$ of IR-780); (C) the typical thermal images of IPM, free IR-780 in PBS (pH 7.4) $(0.15 \mathrm{mg} / \mathrm{mL}$ of IR-780) and saline control upon $808 \mathrm{~nm}$ laser irradiation; (D) the in vitro temperature variations of IPM, free IR-780 in PBS (pH 7.4) $(0.15 \mathrm{mg} / \mathrm{mL}$ of IR-780) and saline control upon $808 \mathrm{~nm}$ laser irradiation. 
the NIR region, at a level comparable to that of free IR-780 in methanol (Figure 2B). Then, the in vitro heat-generating capacity of IPMs was determined after exposure to $808 \mathrm{~nm}$ laser irradiation (Figures 2C and 2D). The typical thermographic images of IPMs after laser irradiation are shown in Figure 2C. After $808 \mathrm{~nm}$ laser irradiation, the IPMs temperature was clearly increased to $52.6 \pm 1.3^{\circ} \mathrm{C}$, a temperature much higher than that for free IR-780 in PBS (pH 7.4) and the saline control (Figure 2D). These results effectively verified the efficient heat-generating capability of IPMs with NIR laser irradiation. Thus IPMs holding great potential for further in vivo PTT applications.

\section{In vivo targeting to lymphatic metastasis in breast cancer}

To evaluate the in vivo targeting capability of IPMs to tumor sites and metastatic lymph nodes, the in vivo distribution was evaluated in a metastatic breast cancer model (Figure 3A). The 4T1 murine cells, one of the most aggressive metastatic breast cancer cell lines, were used to develop the metastatic breast cancer model ${ }^{[29-32]}$. Free IR-780 easily precipitated in PBS (pH 7.4) at $0.15 \mathrm{mg} / \mathrm{mL}$ (as shown in Figure 2A), thus preventing its applicability for various in vivo measurements. The captured images showed that the fluorescence signals were present in the feet with inoculation of 4T1 cancer cells, but they were not detected in the control feet without tumor inoculation. Moreover, the fluorescence intensity in the tumor gradually increased with time and peaked at $4.0 \mathrm{~h}$ after injection. Then, mice were autopsied at $4.0 \mathrm{~h}$ after injection, and the major organs were carefully collected for detection under an in vivo imaging system (Figure 3B). The fluorescence signals were readily observed in the lungs, kidneys, tumor and lymph nodes with high intensity, thus confirming the good targeting ability of IPMs to the tumor and metastatic lymph node sites. By contrast, no fluorescence signals were detected in the control group. These images verified the efficient targeting of IPMs to the primary tumor and metastatic lymph node sites.

\section{Deep penetration of IPMs in tumor and metastatic lymph nodes}

The deep penetration of IPMs into tumor and metastatic lymph nodes was further measured by LCSM (Figure 4). IPMs are indicated by red fluorescence signals in the captured images. By contrast, the nuclei are visible in blue, and actin are shown as green signals. In both tumor and metastatic lymph nodes, the red fluorescence signals were extensively observed in the exterior and interior regions of these tissues (Figure 4). As shown in Figure 1C and 1D, the hydrophobic IR-780 was strongly entrapped in IPMs with high encapsulation efficiency and exhibited good stability in various physiological solutions, including water, PBS ( $\mathrm{pH}$ 7.4) and PBS (pH 7.4) with $10 \%$ FBS within $24 \mathrm{~h}$. IPMs were observed as micelles mainly in the tumor sites or metastatic lymph nodes at 4.0 after injection. These results suggested that the small IPMs penetrate into the deep inner sides of the primary tumor or metastatic lymph nodes, thus suggesting that they may achieve therapeutic efficacy.
A

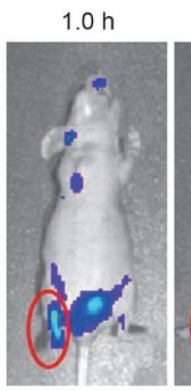

$4.0 \mathrm{~h}$
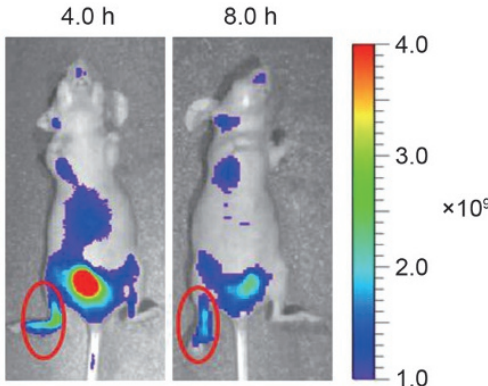

B

Heart Liver Spleen Lung Kidney Tumor Lymph

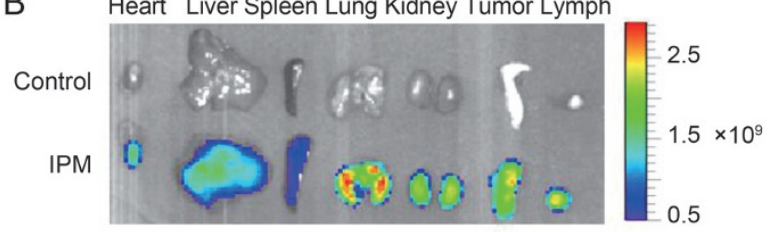

Figure 3. The in vivo distribution of IPM in lymph metastatic breast cancer model, which was induced by injecting 4T1 cancer cells to the footpad of mice. (A) The in vivo fluorescence imaging of IPM at predetermined time intervals after injection; (B) the ex vivo distribution of IPM in each major organ at $4.0 \mathrm{~h}$ after intravenous administration.

\section{In vivo therapeutic efficacy toward lymphatic metastasis in breast cancer}

The in vivo photothermal efficiency of IPMs was evaluated in a lymph metastatic breast cancer model. The thermographic images and temperature changes in tumor tissues were recorded with an infrared thermal imaging camera (Figure $5 \mathrm{~A})$. After NIR laser irradiation, the tumor surface temperature was markedly increased to $57.4 \pm 2.8^{\circ} \mathrm{C}$ in the IPMs+Laser treated group, but it was slightly enhanced to $42.6 \pm 0.7^{\circ} \mathrm{C}$ in the saline+Laser group (Figure 5B). IPMs induced a high photothermal efficiency in vivo after exposure to NIR laser irradiation, thus indicating that they hold great promise in achieving in vivo PTT.

Then, the effectiveness of IPMs in inhibiting tumor growth and lymphatic metastasis of breast cancer was determined. The tumor-bearing mice were administered a single treatment with saline control, saline+Laser, IPMs or IPMs+Laser. Given the in vivo biodistribution profiles in Figure 3, the tumorbearing mice were exposed to laser irradiation $4.0 \mathrm{~h}$ after injection. At d 6 after irradiation, mice were autopsied, and the primary tumor in the foot and metastatic lymph nodes near the primary tumor were carefully removed and weighed to calculate the inhibition rate (Figure 6). Typical photographs of primary tumor and metastatic lymph nodes are shown in Figure $6 \mathrm{~A}$ and $6 \mathrm{C}$. The weight ratio of primary tumors or metastatic lymph nodes from various groups was compared to that of the saline control (Figure 6B and 6D). Compared with the saline control, treatment with the laser alone and IPMs alone had no inhibitory effects on the tumor growth, but the IPMs+Laser treatment resulted in a $70 \%$ inhibition of tumor growth. Moreover, the lymph node weight was used to characterize the inhibitory effects on lymphatic metastasis ${ }^{[19,33,34]}$. The laser alone or IPMs alone had minimal inhibitory effects 


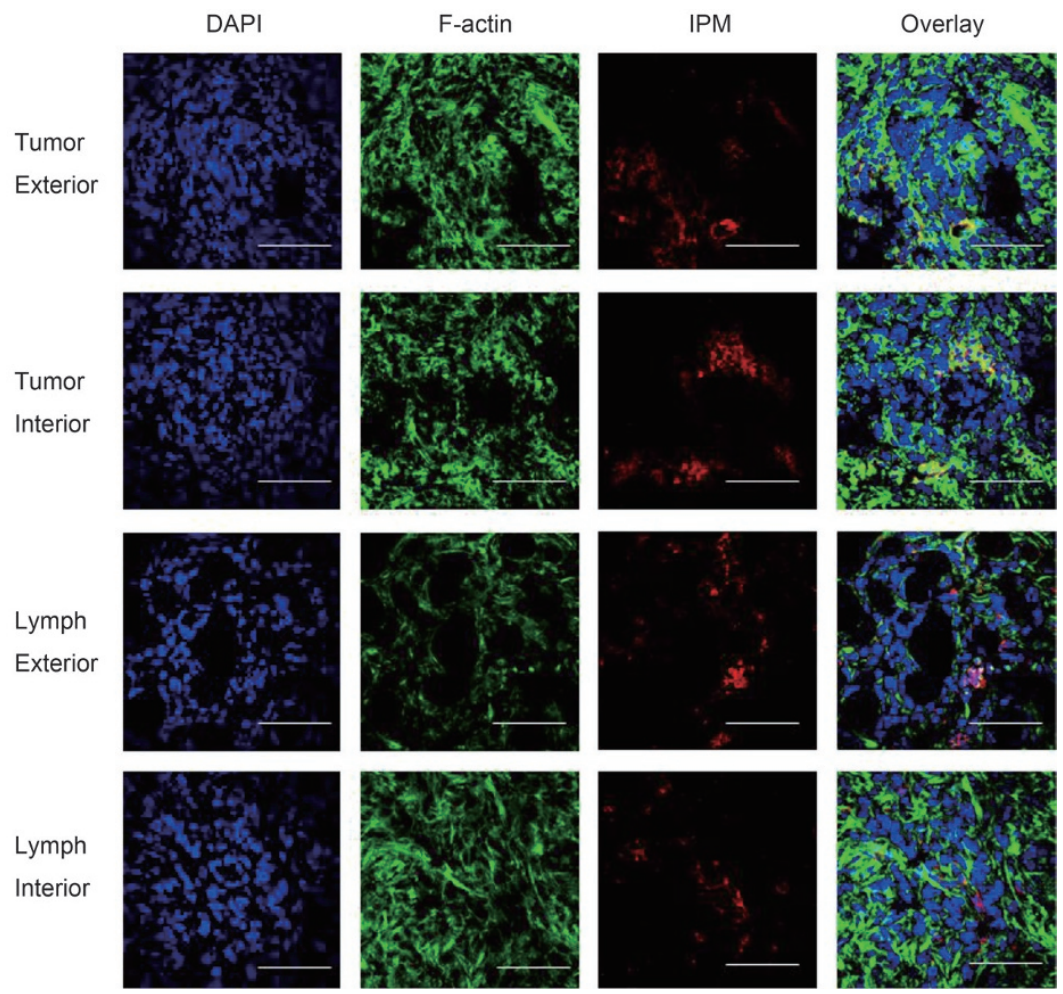

Figure 4. The penetration of IPM in tumor and metastatic lymph nodes detected under LCSM. The detection was performed at $4.0 \mathrm{~h}$ after the injection. The nuclei were stained with DAPI (blue) while the actins were counterstained with phalloidin-FITC (green), scale bar=50 $\mu \mathrm{m}$.

A

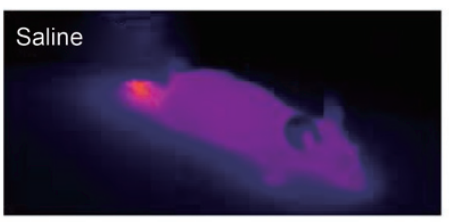

IPM

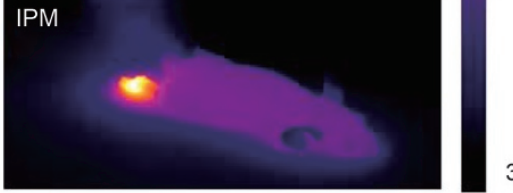

35.7

B

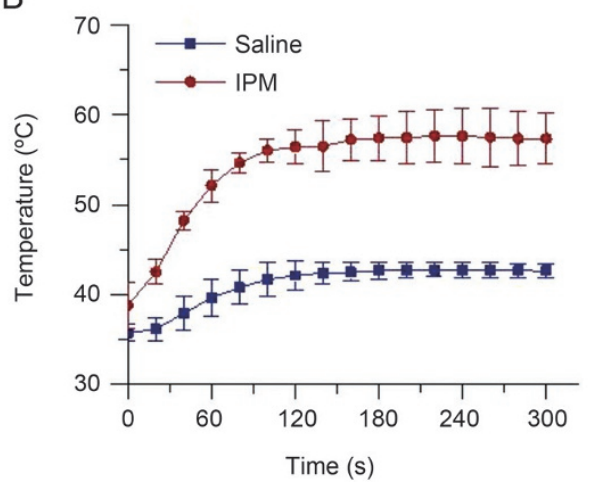

Figure 5. The in vivo photothermal profiles of IPM. (A) The typical thermal images of tumor bearing mice upon $808 \mathrm{~nm}$ laser irradiation; (B) the temperature elevation profiles of tumor tissues in mice treated with saline and IPM. on the lymph node metastasis, but the single IPMs+Laser treatment produced $88.2 \%$ inhibition of lymphatic metastasis. Moreover, lymphatic metastasis was determined by histological examinations of metastatic lymph nodes by H\&E staining. The metastatic lesions in lymph nodes presented as cell clusters with darkly stained nuclei. As shown in Figure 6E, the metastatic foci were clearly detected in the saline control, laser alone and IPMs alone groups, but they were scarcely observed in IPMs+Laser group, thus suggesting considerable inhibition of IPMs+Laser treatment on lymphatic metastasis of breast cancer. As a result, the IPMs-mediated PTT had efficient therapeutic efficacy toward tumor growth and lymph node metastasis of breast cancer, possibly as a result of the high in vitro and in vivo photothermal efficiency of IPMs with NIR laser irradiation, specific tumor targeting and their deep penetrating capability.

\section{Conclusions}

In summary, we developed IPMs with efficient tumor and lymph targeting abilities for potential PTT of lymphatic metastasis in breast cancer. The IPMs are homogeneous nanometersized particles with a mean diameter of $25.6 \mathrm{~nm}$, and they exhibit high heat-generating capability under laser irradiation. In a lymph metastatic breast cancer model, the intravenously injected IPMs accumulated in the tumor and metastatic lymph nodes and penetrated into these tissues. Moreover, a single treatment of IPMs plus laser irradiation significantly inhibited tumor growth and lymphatic metastasis in breast cancer mod- 
A

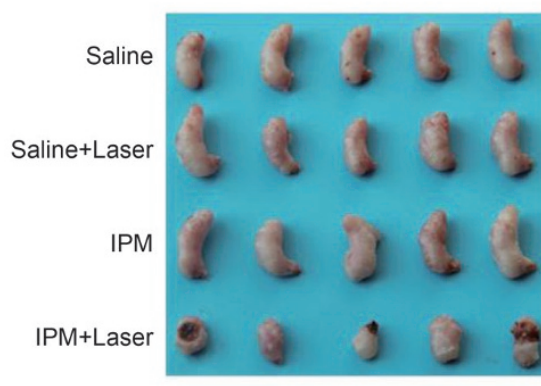

C

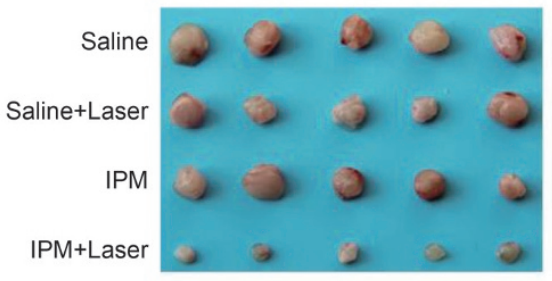

E
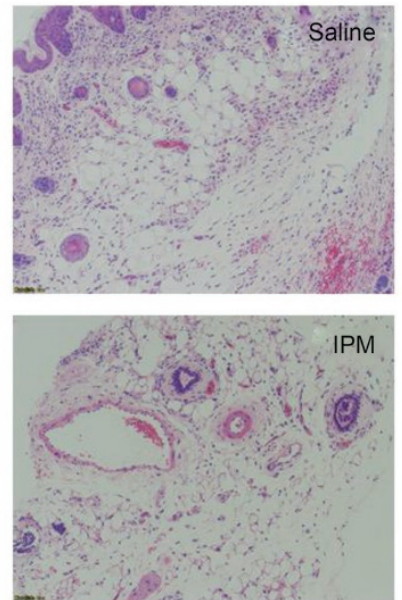

B

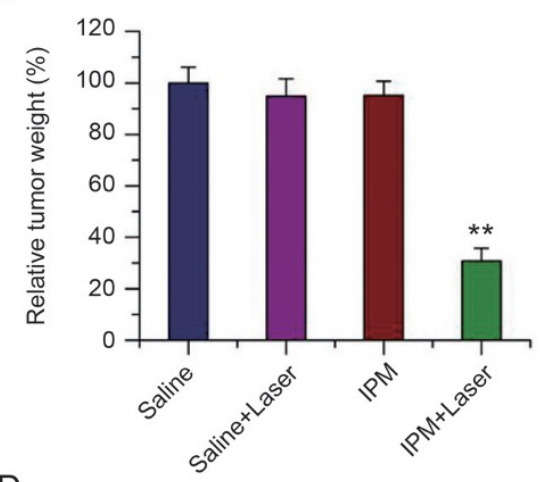

D

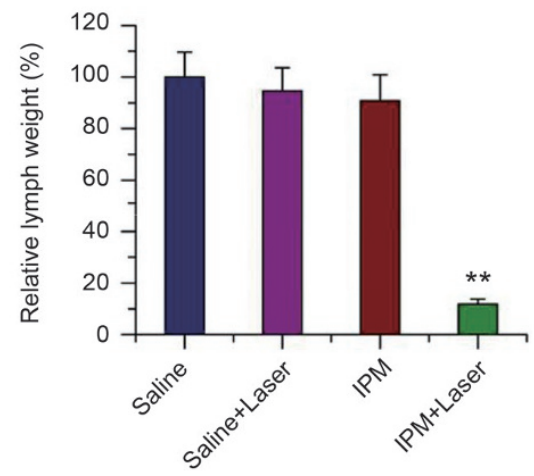

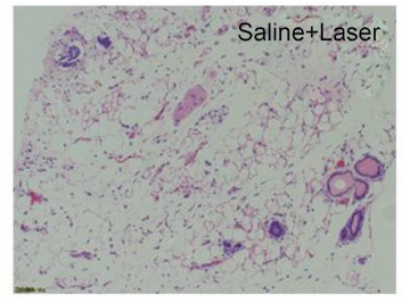

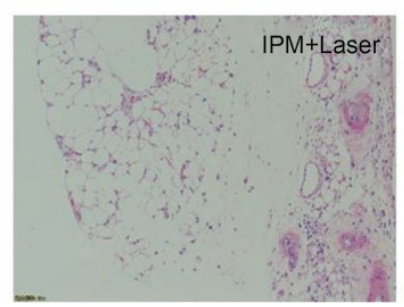

Figure 6. The in vivo therapeutic efficacy of IPM on primary tumor growth and lymph nodes metastasis of breast cancer. (A) The typical images of primary tumor tissues from each group; (B) the relative tumor weight from each group, ${ }^{* *} \mathrm{P}<0.01$; (C) the typical images of metastatic lymph nodes from each group; (D) the relative weight of metastatic lymph nodes from each group, ${ }^{* *} P<0.01$; (E) the histological examinations of the metastatic lymph nodes by H\&E staining.

els. Our findings suggest that IPMs may be an encouraging drug delivery platform for targeting and for PTT applications in metastatic breast cancer.

\section{Acknowledgements}

This work was supported by the National Basic Research Program of China (2013CB932503 and 2015CB932103), National Natural Science Foundation of China (81521005), Institutes for Drug Discovery and Development of CAS (CASIMM0120163005) and Youth Innovation Promotion Asso- ciation of CAS.

\section{Author contribution}

Bin HE performed most of the experiments and drafted the manuscript; Hai-yan HU and Tao TAN assisted in cell culture and in vivo evaluations in tumor bearing mice; Hong WANG participated in cell culture; Kao-xiang SUN and Ya-ping LI revised the manuscript; Zhi-wen ZHANG designed the research and revised the manuscirpt. All authors reviewed the manuscript. 


\section{References}

1 Steeg PS. Targeting metastasis. Nat Rev Cancer 2016; 16: 201-18.

2 van Denderen BJ, Thompson EW. Cancer: The to and fro of tumour spread. Nature 2013; 493: 487-8.

3 Wan LL, Pantel K, Kang YB. Tumor metastasis: moving new biological insights into the clinic. Nat Med 2013; 19: 1450-64.

4 Cao H, Dan Z, He X, Zhang Z, Yu H, Yin Q, et al. Liposomes coated with isolated macrophage membrane can target lung metastasis of breast cancer. ACS Nano 2016; 10: 7738-48.

5 Tong ZS, Miao PT, Liu TT, Jia YS, Liu XD. Enhanced antitumor effects of BPD-MA-mediated photodynamic therapy combined with adriamycin on breast cancer in mice. Acta Pharmacol Sin 2012; 33: 1319-24.

6 Schroeder A, Heller DA, Winslow MM, Dahlman JE, Pratt GW, Langer $\mathrm{R}$, et al. Treating metastatic cancer with nanotechnology. Nat Rev Cancer 2011; 12: 39-50.

7 Chen Q, Liang C, Wang X, He J, Li Y, Liu Z. An albumin-based theranostic nano-agent for dual-modal imaging guided photothermal therapy to inhibit lymphatic metastasis of cancer post surgery. Biomaterials 2014; 35: 9355-62.

8 Liang C, Diao S, Wang C, Gong H, Liu T, Hong G, et al. Tumor metastasis inhibition by imaging-guided photothermal therapy with single-walled carbon nanotubes. Adv Mater 2014; 26: 5646-52.

9 Sahai E. Illuminating the metastatic process. Nat Rev Cancer 2007; 7: 737-49.

10 Yan J, Hua F, Liu HZ, Yang HZ, Hu ZW. Simultaneous TLR2 inhibition and TLR9 activation synergistically suppress tumor metastasis in mice. Acta Pharmacol Sin 2012; 33: 503-12.

11 He XY, Bao XY, Cao HQ, Zhang ZW, Yin Q, Gu WW, et al. Tumorpenetrating nanotherapeutics loading a near-Infrared probe inhibit growth and metastasis of breast cancer. Adv Funct Mater 2015; 25 : 2831-9.

12 Zou L, Wang H, He B, Zeng L, Tan T, Cao H, et al. Current approaches of photothermal therapy in treating cancer metastasis with nanotherapeutics. Theranostics 2016; 6: 762-72.

$13 \mathrm{Cao} \mathrm{H}$, Zou L, He B, Zeng L, Huang Y, Yu H, et al. Albumin biomimetic nanocorona improves tumor targeting and penetration for synergistic therapy of metastatic breast cancer. Adv Funct Mater 2017; Doi: 10.1002/adfm.201605679.

14 Tammela T, Saaristo A, Holopainen T, Yla-Herttuala S, Andersson LC, Virolainen S, et al. Photodynamic ablation of lymphatic vessels and intralymphatic cancer cells prevents metastasis. Sci Transl Med 2011; 3: 69ra11.

15 Sun HP, Su JH, Meng QS, Yin Q, Zhang ZW, Yu HJ, et al. Silibinin and indocyanine green-loaded nanoparticles inhibit the growth and metastasis of mammalian breast cancer cells in vitro. Acta Pharmacol Sin 2016; 37: 941-9.

16 Lim ZZ, Li JE, Ng CT, Yung LY, Bay BH. Gold nanoparticles in cancer therapy. Acta Pharmacol Sin 2011; 32: 983-90.

17 Liu JP, Wang TT, Wang DG, Dong AJ, Li YP, Yu HJ. Smart nanoparticles improve therapy for drug-resistant tumors by overcoming pathophysiological barriers. Acta Pharmacol Sin 2017; 38: 1-8.

18 Wang S, Zhang Q, Luo XF, Li J, He H, Yang F, et al. Magnetic graphene-based nanotheranostic agent for dual-modality mapping guided photothermal therapy in regional lymph nodal metastasis of pancreatic cancer. Biomaterials 2014; 35: 9473-83.

19 Wang S, Zhang Q, Yang P, Yu X, Huang LY, Shen S, et al. Manganese oxide-coated carbon nanotubes as dual-modality lymph mapping agents for photothermal therapy of tumor metastasis. ACS Appl Mater Interfaces 2016; 8: 3736-43.

20 Huo D, He J, Li H, Huang AJ, Zhao HY, Ding Y, et al. X-ray CT guided fault-free photothermal ablation of metastatic lymph nodes with ultrafine HER-2 targeting W18049 nanoparticles. Biomaterials 2014; 35: 9155-66.

21 Wang H, Shen J, Li Y, Wei Z, Cao G, Gai Z, et al. Magnetic iron oxidefluorescent carbon dots integrated nanoparticles for dual-modal imaging, near-infrared light-responsive drug carrier and photothermal therapy. Biomater Sci 2014; 2: 915-23.

22 Chen Y, Li Z, Wang H, Wang Y, Han H, Jin Q, et al. IR-780 loaded phospholipid mimicking homopolymeric micelles for near-IR imaging and photothermal therapy of pancreatic cancer. ACS Appl Mater Interfaces 2016; 8: 6852-8.

23 Yan F, Duan W, Li Y, Wu H, Zhou Y, Pan M, et al. NIR-laser-controlled drug release from DOX/IR-780-loaded temperature-sensitiveliposomes for chemo-photothermal synergistic tumor therapy. Theranostics 2016; 6: 2337-51.

24 Kuang Y, Zhang K, Cao Y, Chen X, Wang K, Liu M, et al. Hydrophobic IR-780 dye encapsulated in CRGD-conjugated solid lipid nanoparticles for NIR imaging-guided photothermal therapy. ACS Appl Mater Interfaces 2017; 9: 12217-26.

25 Cabral H, Matsumoto Y, Mizuno K, Chen Q, Murakami M, Kimura M, et al. Accumulation of sub-100 nm polymeric micelles in poorly permeable tumours depends on size. Nat Nanotechnol 2011; 6: 815-23.

26 Schädlich A, Caysa H, Mueller T, Tenambergen F, Rose C, Göpferich A, et al. Tumor accumulation of NIR fluorescent PEG-PLA nanoparticles: impact of particle size and human xenograft tumor model. ACS Nano 2011; 5: 8710-20.

27 Duan X, Li Y. Physicochemical characteristics of nanoparticles affect circulation, biodistribution, cellular internalization, and trafficking. Small 2013; 9: 1521-32.

28 Bertrand N, Leroux JC. The journey of a drug-carrier in the body: an anatomo-physiological perspective. J Control Release 2012; 161 : 152-63.

29 Qin L, Zhang F, Lu X, Wei X, Wang J, Fang X, et al. Polymeric micelles for enhanced lymphatic drug delivery to treat metastatic tumors. J Control Release 2013; 171: 133-42.

30 Cao HQ, Zhang ZW, Zhao S, He XY, Yu HJ, Yin Q, et al. Hydrophobic interaction mediating self-assembled nanoparticles of succinobucol suppress lung metastasis of breast cancer by inhibition of VCAM-1 expression. J Control Release 2015; 205: 162-71.

31 Zhang ZW, Cao HQ, Jiang SJ, Liu ZY, He XY, Yu HJ, et al. Nanoassembly of probucol enables novel therapeutic efficacy in the suppression of lung metastasis of breast cancer. Small 2014; 10: 4735-45.

$32 \mathrm{He} \mathrm{XY,} \mathrm{Yu} \mathrm{HJ,} \mathrm{Bao} \mathrm{XY,} \mathrm{Cao} \mathrm{HQ,} \mathrm{Yin} \mathrm{Q,} \mathrm{Zhang} \mathrm{ZW,} \mathrm{et} \mathrm{al.} \mathrm{pH-responsive}$ wormlike micelles with sequential metastasis targeting inhibit lung metastasis of breast cancer. Adv Healthc Mater 2016; 5: 439-48.

33 Cabral H, Makino J, Matsumoto Y, Mi P, Wu HL, Nomoto T, et al. Systemic targeting of lymph node metastasis through the blood vascular system by using size-controlled nano carriers. ACS Nano 2015; 9: 4957-67.

34 Xiao J, Duan X, Yin Q, Miao Z, Yu H, Chen C, et al. The inhibition of metastasis and growth of breast cancer by blocking the NF-KB signaling pathway using bioreducible PEl-based/p65 shRNA complex nanoparticles. Biomaterials 2013; 34: 5381-90. 